\title{
ESTUDIO PALEOBOTÁNICO, RECONSTRUCCIÓN PALEOAMBIENTAL Y ASPECTOS TAFONÓMICOS DEL AFLORAMIENTO VALLESIENSE DE COLL DE SAIG (LA CERDAÑA, LÉRIDA, ESPAÑA)
}

\author{
Eduardo BARRÓN
}

Departamento de Geociências. Universidade de Aveiro. 3180 Aveiro (Portugal)

Barrón, E. 1999. Estudio paleobotánico, reconstrucción paleoambiental y aspectos tafonómicos del afloramiento vallesiense de Coll de Saig (La Cerdaña, Lérida, España). [Palaeobotanical study, palaeoenvironmental reconstruction and taphonomical aspects of the vallesian outcrop of Coll de Saig (La Cerdaña, Lérida, Spain)]. Revista Española de Paleontología, n⿳0 extr. Homenaje al Prof. J. Truyols, 77-88. ISSN 0213-6937.

\begin{abstract}
Palynomorphs (36 taxa) and plant megafossils (mainly leaf remains) belonging to 49 species have been identified from Vallesian strata at Coll de Saig in the Cerdaña region of Lleida, Catalonia, Spain. They belong to the divisions Equisetophyta, Pteridophyta, Pinophyta and Magnoliophyta. Although the palynological assemblages are very poor due to the destruction of pollen as a result of the taphonomic characteristics of the outcrop, it has been possible to infer the existence of temperate mesophytic forests with predominant Pinaceae, Betulaceae, Fagaceae and Ulmaceae. On the other hand, the study of plant megafossils has allowed concluding on a hottemperate or subtropical climatic conditions with a high humidity index similar to that found today in eastern Asia. This permitted the development of lauroid evergreen forests dominated by Fagaceae and Lauraceae, and mixed mesophytic forests.
\end{abstract}

Keywords: Palaeobotany, palaeoecology, taphonomy, palynomorphs, megaremains, Vallesian, Upper Miocene, Coll de Saig, La Cerdaña, Spain.

\section{RESUMEN}

Se realiza un estudio paleobotánico (micro y macrorrestos) del afloramiento vallesiense de Coll de Saig (la Cerdaña, Lérida, España) tras el cual se han identificado 36 taxones a partir de palinomorfos y 49 a partir de macrorrestos (fundamentalmente restos foliares), pertenecientes a las divisiones Equisetophyta. Pteridophyta, Pinophyta y Magnoliophyta. El estudio esporopolínico revela unas asociaciones muy pobres en número de ejemplares debido a destrucción polínica a causa de las características tafonómicas del afloramiento, infiriéndose la existencia de bosques mesofíticos templados donde dominaban géneros de las familias Pinaceae. Betulaceae, Fagaceae y Ulmaceae. Por otra parte, el estudio macroflorístico pone en evidencia unas condiciones climáticas de tipo templado cálido o subtropicales, semejantes a las que hoy existen en el este de Asia, con un alto índice de humedad que permitió el desarrollo de laurisilvas, dominadas por Fagaceae y Lauraceae, y bosques mixtos mesofíticos.

Palabras clave: Paleobotánica, paleoecología, tafonomía, palinomorfos, macrorrestos, Vallesiense, Mioceno Superior, Coll de Saig, la Cerdaña, España.

\section{INTRODUCCIÓN Y ANTECEDENTES}

Realizar un estudio paleoflorístico integral de un afloramiento es inusual ya que la mayoría de las veces se abordan por separado el estudio de los macro y de los microrrestos. Nosotros pensamos, al igual que Chaloner (1968), que no es más completa una micro que una macroflora o viceversa, sino que cada una de ellas dará una imagen incompleta de la vegetación que existió en el pasado en una determinada región. Así, como expresan Leroy y Roiron (1996), existen muchas ventajas al estudiarlas de forma conjunta ya que la macroflora nos da una visión local de la vegetación a nivel específico, mientras que la microflora lo hace a nivel local, regional y en menor medida extrarregional.

El caso del afloramiento del Coll de Saig (comarca de la Cerdaña, provincia de Lérida) a nuestro entender es excepcional, ya que sus sedimentos presentan un elevado 


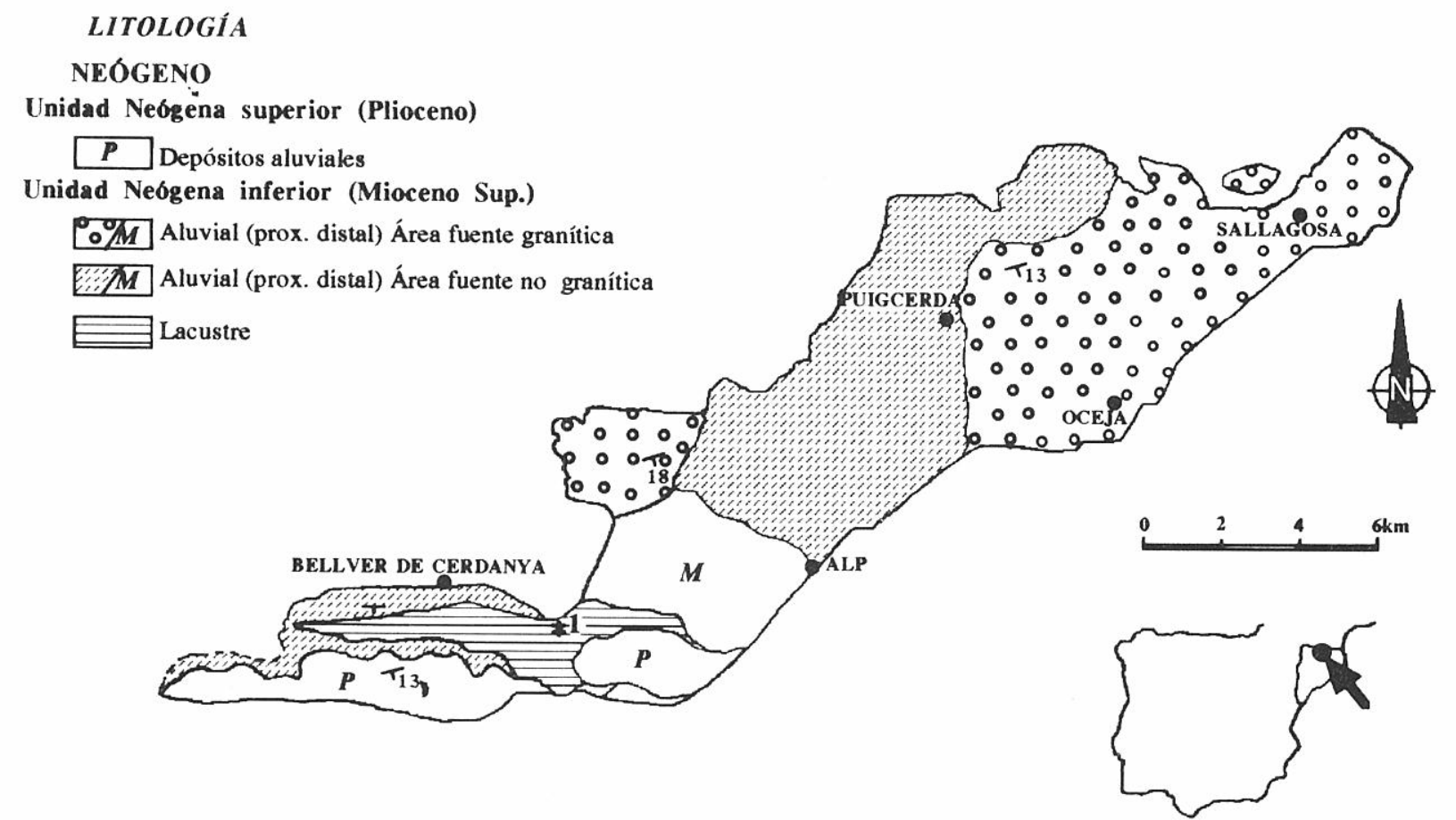

Figura 1. Esquema geológico de la cuenca de la Cerdaña, modificado de Agustí y Roca (1987). 1: Coll de Saig.

número de restos macroscópicos vegetales, fundamentalmente restos foliares y frutos alados y, en menor medida, palinomorfos.

La importancia de este trabajo radica en reunir datos paleobotánicos, tanto palinológicos como macroflorísticos, y tratar de correlacionarlos entre sí para obtener conclusiones de tipo paleoecológico y tafonómico.

Aunque los primeros trabajos paleobotánicos que se realizaron en la Cerdaña datan de finales del siglo pasado (Rérolle, 1884-1885), nunca se expuso la localización de los afloramientos de donde se obtuvieron los macrorrestos vegetales estudiados.

Coll de Saig, como localidad fosilífera, fue descrito por Villalta y Crusafont (1945), quienes lo ubicaron "en las mismas márgenes de la carretera" (de Bellver de Cerdanya a Prats). Según los autores citados, junto con el afloramiento que denominan "Badés", Coll de Saig es uno de los puntos en donde se recolectan mejores ejemplares macroscópicos ya que presenta arcillas muy homogéneas y de finísimo grano que se exfolian con facilidad. En este afloramiento determinaron una larga lista de restos vegetales que atribuyeron a 28 taxones. Una parte de los ejemplares estudiados por Villalta y Crusafont (op. cit.) están integrados hoy en día en las colecciones del Instituto Tecnológico Geominero de España y fueron utilizados en la memoria explicativa de la hoja de Bellver (escala 1.50.000, hoja 216) realizada por Solé Sabarís y Llopis Lladó (1947); la otra parte integra la colección J.F. Villalta que se conserva en el Museo de Geología de Barcelona.

Posteriormente, otros autores, como Menéndez Amor (1955), cuya colección se encuentra depositada en el Museo Nacional de Ciencias Naturales (CSIC), y Álvarez Ramis y Golpe-Posse (1981), estudiaron restos macroscópicos procedentes de este afloramiento considerando todos los taxones citados por Villalta y Crusafont (1945) y aumentando en más de 50 la lista elaborada por estos.

En los últimos años, Coll de Saig fue estudiado desde un punto de vista tafonómico (Barrón, 1993; Martín Closas, 1995), deduciéndose a partir de los macrorrestos las condiciones de fosilización que existieron en el medio lacustre de la zona.

Hasta el momento no se habían realizado estudios palinológicos de los materiales de Coll de Saig, ya que los que se han llevado a cabo en la Cerdaña se circunscribieron a afloramientos de facies de abanicos aluviales (Jelgersma, 1957; Bessedik, 1985; Baltuille et al., 1992; Barrón, 1996b, 1997), y no a los de fondo de lago. Por esta razón, este trabajo es también una contribución al conocimiento palinológico de las facies de fondo de lago de la cuenca de la Cerdaña.

\section{SITUACIÓN GEOGRÁFICA Y GEOLÓGICA}

La Cerdaña es una antigua cuenca lacustre situada en los Pirineos orientales, entre Francia y España (Fig. 1). Actualmente constituye una llanura alargada de $25-30 \mathrm{~km}$ de longitud por 5-7 de ancho, cruzada en su parte norte por el río Segre, que se encuentra a unos $1.100 \mathrm{~m}$ sobre el nivel del mar.

Esta cuenca fue formada durante los movimientos dextrales NE-SO de la Falla de la Tet (Cabrera et al., 1988). Julià (1984) diferencia dos cubetas: la de Bellver, con una importante etapa lacustre profunda, y la de Sanavastre, con una fuerte influencia fluvial. 
El relleno sedimentario de la Cerdaña está integrado por potentes sucesiones detríticas y se organiza en dos grandes unidades estratigráficas (Fig. 1): la Unidad Neógena Inferior (Vallesiense medio-superior) formada por la interacción de procesos aluviales y lacustres; y la Unidad Neógena Superior (post-Vallesiense), constituida exclusivamente por depósitos aluviales (Roca, 1986).

En 1989, Anadón et al. describieron una columna estratigráfica sintética de la Unidad Neógena Inferior de los materiales de la Cerdaña, estableciendo la siguiente sucesión ubicada sobre un basamento de sedimentos paleozoicos (A) (Fig. 2):

B- Asociación de terrígenos: consistente en gravas de color marrón pálido, amarillento y grisáceo, arenas arcósicas, mudstones y lechos de lignitos, con más de 110 $\mathrm{m}$ de espesor. Localmente aparecen niveles de lignitos poco potentes.

C-Asociación diatomítica: compuesta por más de 250 $\mathrm{m}$ de espesor de diatomitas y mudstones de color grisazulado o gris pálido, con gran cantidad de materia orgánica. En esta unidad aparecen láminas intermedias de arenas con ostrácodos. Algunos de los niveles son ricos en $\mathrm{CaPO}_{4}$ y $\mathrm{FePO}_{4}$, o poseen nodulos aislados. Las facies diatomíticas presentan una fina laminación que puede ser relacionada con varvas estacionales de sedimentación.

D- Asociación superior de terrígenos: está formada por mudstones, cuyo color va de gris a marrón, areniscas y conglomerados con unos $100 \mathrm{~m}$ de grosor. En este tramo también se registran niveles de lignitos.

El afloramiento de Coll de Saig está situado en la cubeta de Bellver y en el tramo $\mathrm{C}$ de la columna estratigráfica. Más concretamente, se encuentra en el margen izquierdo de la carretera de Bellver de Cerdanya a Prats (carretera comarcal 1.411), en esa dirección. Latitud: $42^{\circ} 22^{\prime} 2^{\prime}$ 'N. Longitud: $1^{\circ} 49^{\prime} 20^{\prime}$ 'O. Presenta 2-1,5 m de potencia, estando constituido por arcillas varvadas amarillentas y anaranjadas.

Coll de Saig ha sido incluido a partir de datos tafonómicos en una zona lacustre litoral a donde llegaban macrorrestos vegetales transportados por corrientes de agua y vientos (Martín Closas, 1995). El autor citado indica que la cercanía a la orilla se deduce por la conservación de organismos bentónicos, tales como plantas acuáticas y ostrácodos. Nosotros no hemos observado ostrácodos en los sedimentos del afloramiento, aunque se encuentran abundantemente en otros puntos de la zona, como son San Salvador y el torrente de Vilella. Por su parte, la presencia de plantas acuáticas, como Potamogeton, es también un hecho en afloramientos propios de facies lacustres distales, como ocurre en el barranco de Salanca (Barrón, 1996a). Considerando las razones expuestas, la ubicación de Coll de Saig en una zona lacustre cercana a la orilla debiera ser revisada.

\section{MATERIAL Y MÉTODOS}

En este afloramiento se han recogido de base a techo 7 muestras de arcillas y diatomitas amarillentas y anaranjadas para realizar análisis polínicos (CS1-CS7), y

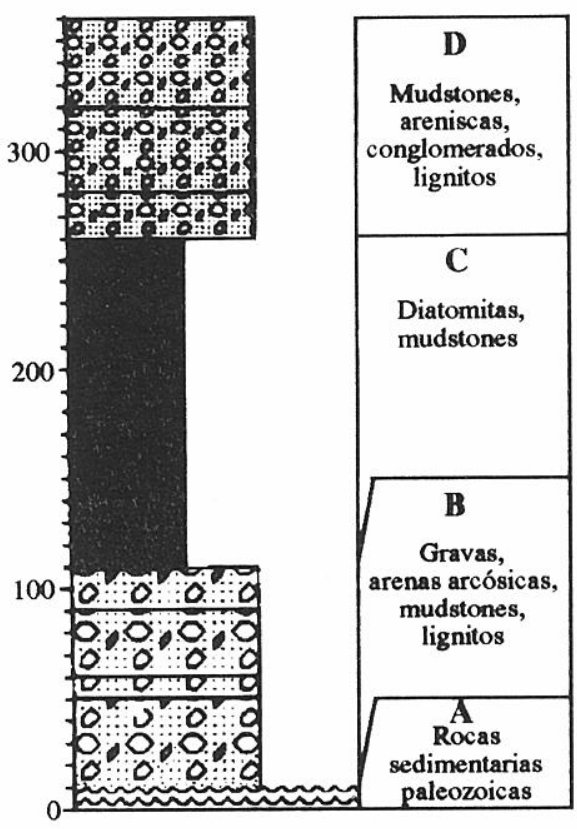

Figura 2. Columna estratigráfica sintética de la Unidad Neógena Inferior de la cuenca de la Cerdaña, modificada de Anadón et al. (1989).

gran cantidad de restos vegetales macroscópicos. Las muestras polínicas se recogieron respectivamente a 0,35 , $50,70,85,103$ y $118 \mathrm{~cm}$ de la base. Como ya hemos indicado, todas ellas proceden del tramo C de la sucesión propuesta por Anadón et al. (1989) (Fig. 2). No ha sido posible conocer su posición estratigráfica precisa dentro de dicho tramo por problemas de exposición.

La separación de los palinomorfos fue llevada a cabo usando las técnicas descritas por Phipps y Playford (1984), basadas en tratamiento ácido $\left(\mathrm{HCl}, \mathrm{HF}\right.$ y $\left.\mathrm{HNO}_{3}\right)$; y su concentración final fue estimada tras pasar el residuo resultante a través de tamices de 500, 250, 75, 50 y 12 $\mu \mathrm{m}$. Tras el montaje de las placas, éstas se estudiaron con la ayuda de un Microscopio óptico LEITZ LABORLUX D.

Para que la muestra fuera estadísticamente representativa, se consideró como número mínimo de granos de palinomorfos a contar por cada muestra procesada un conjunto entre 500-1.000, como indican Jonker (1951) y Green y Dolman (1988). Los palinomorfos se han determinado fundamentalmente a nivel genérico o familiar, ya que todos ellos pueden ser relacionados con taxones actuales.

Los macrorrestos estudiados son fundamentalmente restos foliares, frutos alados y conos de gimnospermas que aparecen siempre en forma de impresiones y compresiones con y sin restos cuticulares conservados, en el sentido propuesto por Barrón (1993). Estos ejemplares integran las colecciones del Museo Geominero, Museo de Geología de Barcelona, Museo Nacional de Ciencias Naturales (CSIC), Museo del Seminario de Barcelona, Departamento de Paleontología de la Facultad de Ciencias Geológicas de la Universidad Complutense de Madrid; varias colecciones particulares y ejemplares recogidos por nosotros en diferentes campañas de campo. 
Para el estudio de este tipo de fósiles se ha utilizado una lupa binocular WILD M8 con cámara clara acoplada.

Entre los macrorrestos descritos hemos hallado algunas especies con morfologías idénticas a las que presentan algunos taxones actuales, por ejemplo: Fagus gussonii Mass., especie fósil encontrada en Coll de Saig, semejante a $F$. orientalis Lipsky (Barrón y Diéguez, 1994). Sin embargo, como explica Collinson (1986), si usáramos el binomio de la especie actual al determinar estos restos, supondríamos aspectos adicionales de información taxonómica que no nos proporciona el fósil. Además, es posible que un tipo de órgano pueda haberse presentado de forma idéntica en especies diferentes, sin que podamos apreciarlo en el registro fósil. Por estas dos razones, las especies se han descrito siempre de forma parataxónica. A diferencia de las especies, siempre que ha sido posible se ha empleado, como en el caso de los palinomorfos, la nomenclatura genérica actual.

Siguiendo la nomenclatura propuesta por Fernández López (1991), considerada para clasificar el conjunto de las especies determinadas a partir de macrorrestos en la misma Cerdaña por Barrón (1993), los taxones identificados se han agrupado en tres categorías paleobiológicas y tafonómicas:

1- Taxones démicos representados por elementos autóctonos, que corresponden a plantas acuáticas que se desarrollaron en la zona, como Potamogeton y Nymphaeaceae.

2- Taxones démicos representados por elementos alóctonos, representados por las plantas ripícolas, entre las que se pueden destacar: Alnus, Salix, Fraxinus y Carya, entre otras.

3- Taxones adémicos representados por elementos alóctonos, tratándose de las plantas que habitaron en las zonas emergidas de la cuenca que no necesitaron de una humedad edáfica permanente para su desarrollo, como Pinus, Fagus y Quercus.

Para realizar una interpretación paleoclimática a partir de los palinomorfos y macrorrestos, se han clasificado los taxones determinados según los requerimientos térmicos de especies actuales próximas a ellos filogenéticamente en el sentido de Van Steenis (1962), modificado por Bessedik (1985). En el caso del conjunto de los macrorrestos foliares, el clima ha sido deducido a partir de las características fisonómicas de estos, considerándose el tamaño foliar y el tipo de margen según lo expuesto por Bailey y Sinnot $(1915,1916)$, Raunkiaer (1934) y Webb (1959).

\section{ESTUDIO PALINOLÓGICO}

Únicamente dos de las muestras recogidas CS2 y CS6 han sido significativas desde un punto de vista palinológico, según lo expresado en el apartado anterior. Las muestras CS1, CS3, CS5 y CS7 no poseían ningún contenido palinológico, mientras que la CS4 lo presentaba pero no era significativo.

Es de resaltar la baja proporción de palinomorfos que muestran los sedimentos de Coll de Saig si se compara con las de otros afloramientos ceretanos (Barrón, 1996a). Esto es debido a la baja cantidad de materia orgánica de sus materiales. Como expuso Barrón (1993), los afloramientos de fondo de lago de la Cerdaña no se diferencian en su composición mineralógica, fundamentalmente arcillas y diatomitas, sino en el mayor o menor contenido en materia orgánica. La escasez de ésta en Coll de Saig puede ser debida a dos factores: una mayor exposición del afloramiento a las condiciones climáticas, fundamentalmente lluvias que producen lixiviación de la materia orgánica; y/o diferentes procesos fosildiagenéticos debidos al grado de anoxia en algunas partes de las cuenca lacustre.

Las muestras estudiadas presentan unos porcentajes semejantes de pólenes de gimnospermas (Fig. 3), las cuales están representadas mayoritariamente por los géneros Pinus y Abies. Estos dos taxones son los que poseen tantos por ciento más elevados, lo cual seguramente es debido a una sobrerrepresentación, según el concepto de Barrón (1996c), dado su carácter anemófilo. En segundo lugar, es destacable la presencia de otras Pinaceae como Cathaya, Cedrus y Picea, la escasa representación de Cupressaceae, la presencia puntual de Ephedra en la muestra CS6 y la ausencia de polen asignable a la familia Taxodiaceae.

Las angiospermas arbóreas se reunen en un grupo importante de taxones con porcentajes mucho menores que los del conjunto de Pinaceae (Fig. 3), destacándose por sus valores Alnus, Ulmus-Zelkova, Quercus, Fagus y Juglans. De una forma casi puntual se han encontrado Pterocarya, Carpinus, Carya, Betula, Tilia, Salix, Acer y el tipo Robinia. Entre las angiospermas arbustivas destacan Corylus y Myrica, apareciendo testimonialmente Sapotaceae, Ericaceae, Buxus y Cistus. Se ha encontrado un solo grano de polen en la muestra CS2 asignable al género Parthenocissus, el cual representa a las plantas lianoides que existieron en la zona.

Con respecto a las angiospermas no arbóreas (Fig. 3), las mejor representadas son Poaceae con una relativa buena representación en CS6. El resto de los taxones herbáceos aparecen en muy bajas proporciones y en una u otra de las muestras estudiadas, exceptuando a Sparganiaceae-Typhaceae que se encuentra en las dos. Por su parte, las criptógamas poseen una presencia muy baja, reseñándose únicamente el tipo Polypodium en CS2.

Si ahora analizamos el contenido de las dos muestras por separado (Fig. 3), observamos que CS6 posee una proporción más alta de taxones que CS2, lo cual posiblemente sea debido a una menor tasa de destrucción polínica. En CS6 no se ha encontrado el tipo Polypodium, la familia Ericaceae y los géneros Betula, Parthenocissus y Convolvulus. A causa de la escasez de palinomorfos encontrada no podemos asegurar que la ausencia de los taxones mencionados en este nivel sea determinativa de cambios en las condiciones ambientales de la zona.

Los porcentajes totales obtenidos tanto en CS2 como en CS6 señalan que en la zona existieron bosques de coníferas integrados fundamentalmente por Pinus y Abies, y también la presencia de elementos propios de 


\begin{tabular}{|c|c|c|c|c|c|}
\hline \multirow[t]{2}{*}{ TAXONES } & \multicolumn{2}{|c|}{ CS2 } & \multicolumn{2}{|c|}{ CS6 } & \multirow{2}{*}{$\begin{array}{l}\text { REQUERIMIENTOS } \\
\text { TÉRMICOS }\end{array}$} \\
\hline & $\mathbf{N}^{0}$ & $\%$ & $\mathbf{N}^{0}$ & $\%$ & \\
\hline Tipo Polypodium & 1 & 0,06 & - & & Pluri \\
\hline Abies & 314 & 18,88 & 329 & 16,13 & Meso/micro y micro \\
\hline Cathaya & 87 & 5,23 & 149 & 7,31 & Meso \\
\hline Cedrus & 15 & 0,90 & 7 & 0,34 & Meso \\
\hline Picea & 5 & 0,30 & 2 & 0,10 & Meso/micro y micro \\
\hline Pinus & 1.003 & 60,31 & 1.155 & 56,64 & Pluri \\
\hline Pinaceae indet. & - & & 2 & 0,10 & - \\
\hline Cupressaceae & 3 & 0,18 & 3 & 0,15 & Pluri \\
\hline Ephedra & - & & 1 & 0,05 & Meso \\
\hline Ulmus-Zelkova & 13 & 0,78 & 75 & 3,68 & Meso y meso/micro \\
\hline Carya & - & & 3 & 0,15 & Meso \\
\hline Juglans & 3 & 0,18 & 14 & 0,68 & Meso \\
\hline Pterocarya & 1 & 0,06 & 1 & 0,05 & Meso \\
\hline Myrica & 2 & 0,12 & 6 & 0,29 & Meso \\
\hline Fagus & 5 & 0,30 & 17 & 0,83 & Meso/micro \\
\hline Quercus & 12 & 0,72 & 68 & 3,33 & Meso y meso/micro \\
\hline Alnus & 108 & 6,49 & 24 & 1,17 & Meso/micro y micro \\
\hline Betula & 2 & 0,12 & - & & Meso/micro y micro \\
\hline Carpinus & 1 & 0,06 & 2 & 0,10 & Meso/micro \\
\hline Corylus & 7 & 0,42 & 9 & 0,44 & Meso/micro \\
\hline Tilia & - & & 2 & 0,10 & Meso/micro \\
\hline Cistus & - & & 1 & 0,05 & Meso \\
\hline Salix & - & & 1 & 0,05 & Meso/micro \\
\hline Ericaceae & 2 & 0,12 & - & & Pluri \\
\hline Sapotaceae & - & & 1 & 0,05 & Mega y mega/meso \\
\hline Tipo Robinia & 1 & 0,06 & 1 & 0,05 & Meso/micro \\
\hline Parthenocissus & 1 & 0,06 & - & & Meso \\
\hline Buxus & - & & 1 & 0,05 & Meso \\
\hline Acer & - & & 1 & 0,05 & Meso/micro \\
\hline Ranunculus & - & & 2 & 0,10 & $\mathrm{Meso} / \mathrm{micro}$ \\
\hline Amarant.-Chenopodiaceae & - & & 2 & 0,10 & Pluri \\
\hline Convolvulus & 1 & 0,06 & & & Meso/micro \\
\hline Asteraceae tubuliflorae & - & & 2 & 0,10 & Pluri \\
\hline Potamogeton & - & & 1 & 0,05 & Pluri \\
\hline Poaceae & 5 & 0,30 & 26 & 1,27 & Pluri \\
\hline Sparganiaceae-Typhaceae & 2 & 0,12 & 4 & 0,20 & Pluri \\
\hline Indeterminados & 69 & 4,15 & 127 & 6,23 & \\
\hline Totales & 1.663 & 100 & 2.039 & 100 & \\
\hline
\end{tabular}

Figura 3. Espectro polínico del afloramiento de Coll de Saig. Requerimientos térmicos: Mega: megatérmico, Mega/meso: mega-mesotérmico, Meso: mesotérmico, Meso/micro: meso-microtérmico, Micro: microtérmico, Pluri: plurirregional.

formaciones mesofíticas como robledales, hayedos y bosques de ribera y pantano.

Si no consideramos la sobrerrepresentación de Pinaceae, la muestra CS2 destaca por sus valores de taxones démicos representados por elementos alóctonos a causa del porcentaje de Alnus. Esto no ocurre en CS6, en donde las angiospermas terrestres (adémicas representadas por elementos alóctonos), en especial Quercus y Ulmus-Zelkova, poseen unos porcentajes mayores que Alnus. La disminución de este último género y el aumento de los porcentajes de Poaceae en CS6 podría ser indicativo de una apertura de los ecosistemas ribereños.

La mayoría de los taxones determinados corresponden a elementos mesotérmicos y meso/microtérmicos (Fig. 3), indicativos de unas condiciones climáticas de tipo templado. Por su parte, la presencia de Abies con altos porcentajes informa sobre las condiciones montanas de la zona ya que habita siempre en zonas húmedas y frías (Barbéro y Quézel, 1975), y estas condiciones en la 
Península Ibérica durante el Mioceno Superior solamente se hallaban en las regiones montañosas.

\section{ESTUDIO MACROFLORÍSTICO}

Se han estudiado 754 ejemplares, de los cuales el $88,32 \%$ del total (666 ejemplares) corresponden a restos foliares. La diferencia $(11,67 \%)$ corresponde fundamentalmente a pseudofrutos, brácteas, escamas tectrices y conos de gimnospermas y amentos de angiospermas. Es muy baja la proporción de frutos y semillas aladas de angiospermas, 1,89\% del total de restos estudiados.

La mayoría de los ejemplares estudiados se encuentran en forma de impresión $(87,11 \%)$, apareciendo compresiones $(10,45 \%)$ y especímenes con la cutícula conservada $(2,44 \%)$ en un porcentaje muy inferior.

El conjunto de los ejemplares se ha podido atribuir en su totalidad a grandes grupos, siendo el mejor representado el de las angiospermas dicotiledóneas (36 taxones), seguido por gimnospermas (7 taxones), angiospermas monocotiledóneas (4 taxones) y criptógamas vasculares (2 taxones) (Fig. 4). Si ahora consideramos el conjunto de los taxones determinados en este trabajo, se observa un mayor número de citas que las realizadas por Villalta y Crusafont (1945), y un número inferior a las que hicieron Menéndez Amor (1955) y Álvarez Ramis y Golpe-Posse (1981). En referencia al trabajo de Villalta y Crusafont, indicaremos que en nuestro estudio hay un mayor número de citas porque se ha estudiado una muestra mucho más grande que incluye la colección de los autores. Con respecto a los de Menéndez Amor (op. cit.) y Álvarez Ramis y GolpePosse (op. cit.), la reducción en el número de citas se debe a una exhaustiva revisión taxonómica (Barrón, 1996a), que puso de manifiesto la existencia de formas polimórficas en una elevada cantidad de especies que habían sido mencionadas como especies distintas. A modo de ejemplo, expondremos que ejemplares de Acer pyrenaicum Rér. y Alnus occidentalis Rér. fueron asignados respectivamente a 10 especies diferentes (Barrón, 1996d, 1996e).

Por otra parte, hemos de señalar que los autores que estudiaron previamente la macroflora de Coll de Saig citaron especies como Salvinia reussii Ett., Glyptostrobus europaeus (Brong.) Ung. y Pterocarya castaneaefolia (Goepp.) Menz., cuya presencia no ha sido detectada por nosotros.

Tras la revisión taxonómica de la macroflora del afloramiento, ponemos de manifiesto la presencia de taxones que hasta el momento no se habían citado en él, como Cryptomeria rhenana Kilpper (Lám. I, fig. 9), aff. Abies sp., aff. Pinus sp., Tsuga moenana Kirch., Nymphaeaceae, Daphnogene sp. (Lám. I, fig. 5), Laurophyllum sp. (Lám. I, fig. 11), cf. Ocotea sp., Zelkova zelkovaefolia (Ung.) Buz. et Kotl., Myrica lignitum (Ung.) Sap., Myrica marginalis Hr., Fagus gussonii (Lám. I, fig. 10), Quercus mediterranea Ung., aff. Quercus sp., Betula insignis Gaud., Corylus sp.,
Populus tremulaefolia Sap., Salix lavateri $\mathrm{Hr}$, Myrsiniaceae, Fabales (Lám. I, fig. 6), Cornus sp., Acer integerrimum (Viv.) Mass., Acer subcampestre Goepp., Acer sp., aff. Cedrela sp., aff. Aralia sp., Fraxinus numana Mass. (Lám. I, fig. 7), Arecaceae y Commelinidae.

La gran representación de dicotiledóneas arbóreas entre los macrorrestos indica condiciones de baja energía en las aguas del lago, ya que si hubiera habido una alta energía hidráulica los restos foliares de las angiospermas hubieran sido destruidos (Spicer y Wolfe, 1987). Las asociaciones en donde dominan las hojas de angiospermas, como es el caso que nos ocupa, revelan baja velocidad de depósito, lo cual no indica que la comunidad vegetal original no se encontrara dominada por gimnospermas. Esto quiere decir que en la zona debieron existir bosques donde Pinus, Abies, Tsuga, Cryptomeria (Lám. I, fig. 9) y Cupressaceae (Lám. I, fig. 3) tuvieron una buena representación, como lo atestigua la recolecta de acículas, pseudofrutos, piñas, fragmentos de ramas y escamas tectrices. Por otra parte, los helechos como Pteridium oeningense (Ung.) Hantke y las angiospermas monocotiledóneas se encuentran en una baja proporción probablemente por el tipo de abscisión foliar que presentan. Así, la entrada en el medio acuático de sus restos foliares se debió ver dificultada porque al morir estos quedaron aún durante un largo tiempo unidos al tallo de la planta parental (Scheihing y Pfefferkorn, 1984).

Con respecto a las angiospermas dicotiledóneas (Fig. 4), la familia que posee un más alto valor de presencia es Fagaceae, a causa de los altos porcentajes que posee Quercus drymeja Ung. (13,66\%), siendo la especie mejor representada en el afloramiento. Esta quercínea de hojas notófilas (Lám. I, fig. 1) está relacionada con un conjunto de encinas del Extremo Oriente incluidas en el subgénero Cyclobalanus del género Quercus (Barrón, 1998).

La familia Lauraceae es, después de Fagaceae, la que se encuentra en mayor abundancia en Coll de Saig (Fig. 4), hayándose representada por 6 taxones: Daphnogene sp. (Lám. I, fig. 5), Laurophyllum sp. (Lám. I, fig. 11), cf. Ocotea sp., Persea princeps.(Hr.) Sch. (Lám. I, fig. 8) y Lauraceae indeterminadas, todos ellos con hojas notófilas. El estudio de los restos foliares de esta familia es complicado tanto si se aborda desde un punto de vista morfológico, por causa de problemas de anisofilia y polimorfismo, como desde uno cuticular (Ferguson, 1974; Givulescu et al., 1996; Sitár y Kvacek, 1997). Sin embargo, es necesario comparar estos dos aspectos para una perfecta determinación de las especies, lo cual, dado el estado de conservación de los ejemplares estudiados (casi todos ellos impresiones), no ha sido posible. De todas maneras, la primera especie de Lauraceae mencionada, Daphnogene sp. (Lám. I, fig. 5), relacionaría la flora ceretana con la del Extremo Oriente, del mismo modo que $Q$. drymeja, mientras que las tres siguientes lo harían con la Región Macaronésica (Barrón, 1996a). Destaquemos los porcentajes de $P$. princeps $(7,29 \%)$ (Lám. I, fig. 8), especie relacionada filogenéticamente con $P$. indica (I.K.) Spreng., de la Macaronesia. 


\begin{tabular}{|c|c|c|c|c|}
\hline \multirow[t]{2}{*}{ TAXONES } & \multicolumn{2}{|c|}{ MUESTRA ESTUDIADA } & \multirow[t]{2}{*}{ FISONOMÍA } & \multirow{2}{*}{$\begin{array}{l}\text { REQUERIMIENTOS } \\
\text { TÉRMICOS }\end{array}$} \\
\hline & $\mathrm{N}^{\circ}$ & $\%$ & & \\
\hline $\begin{array}{l}\text { cf. Equisetum sp. } \\
\text { Pteridium oeningense (Ung.) Hantke }\end{array}$ & $\begin{array}{l}1 \\
1\end{array}$ & $\begin{array}{l}0,13 \\
0,13\end{array}$ & $\begin{array}{l}\text { Lepto/ liso } \\
\text { Meso/- }\end{array}$ & $\begin{array}{c}\text { Pluri } \\
\text { Meso y meso/micro }\end{array}$ \\
\hline $\begin{array}{l}\text { Cryptomeria rhenana Kilpper } \\
\text { Cupressaceae } \\
\text { aff. Abies sp. (pseudofrutos) } \\
\text { Pinus sp. (acículas) } \\
\text { aff. Pinus sp. (pseudofrutos) } \\
\text { Tsuga moenana Kirch. } \\
\text { Abietoideae }\end{array}$ & $\begin{array}{l}25 \\
2 \\
8 \\
31 \\
3 \\
1 \\
10\end{array}$ & $\begin{array}{c}3,31 \\
0,26 \\
1,06 \\
4,11 \\
0,40 \\
0,13 \\
1,32\end{array}$ & $\begin{array}{l}\text { Lepto/ liso } \\
\text { Lepto/liso } \\
- \\
\text { Lepto/ liso } \\
- \\
- \\
\text { Lepto/liso }\end{array}$ & $\begin{array}{c}\text { Meso } \\
\text { Meso y meso/micro } \\
\text { Meso/micro y micro } \\
\text { Pluri } \\
\text { Pluri } \\
\text { Meso/micro y micro } \\
\text { Meso/micro y micro }\end{array}$ \\
\hline $\begin{array}{l}\text { Nymphaeaceae } \\
\text { Daphnogene sp. } \\
\text { Laurophyllum sp. } \\
\text { cf. Ocotea } \text { sp. } \\
\text { Persea princeps (Hr.) Sch. } \\
\text { Lauraceae indet. } \\
\text { Hamamelidaceae } \\
\text { Ulmus } \text { sp. } \\
\text { Zelkova zelkovaefolia } \text { (Ung.) Buz. et Kotl. } \\
\text { Myrica lignitum (Ung.) Sap. } \\
\text { Myrica marginalis } \text { Hr. } \\
\text { Fagus gussonii Mass. } \\
\text { Fagus pristina Sap. } \\
\text { Fagus sp. (cúpula) } \\
\text { Quercus drymeja Ung. } \\
\text { Quercus hispanica } \text { Rér. } \\
\text { Quercus mediterranea Ung. } \\
\text { aff. Quercus sp. } \\
\text { Alnus occidentalis Rér. } \\
\text { Betula insignis Gaud. } \\
\text { Corylus } \text { sp. } \\
\text { Betulaceae } \\
\text { Tilia vidali } \text { Rér. } \\
\text { Populus tremulaefolia Sap. } \\
\text { Salix lavateri Hr. } \\
\text { Myrsiniaceae } \\
\text { Fabales (restos foliares + legumbres) } \\
\text { Trapa ceretana Rér. } \\
\text { Cornus sp. } \\
\text { Acer pyrenaicum Rér. } \\
\text { Acer integerrimum } \text { (Viv.) Mass. } \\
\text { Acer subcampestre Goepp. } \\
\text { Acer } \text { sp. (flor) } \\
\text { aff. Cedrela } \text { sp. } \\
\text { aff. Aralia } \text { sp. } \\
\text { Fraxinus numana Mass. }\end{array}$ & $\begin{array}{l}1 \\
21 \\
28 \\
1 \\
55 \\
14 \\
7 \\
6 \\
46 \\
35 \\
2 \\
44 \\
12 \\
1 \\
103 \\
61 \\
4 \\
1 \\
60 \\
2 \\
2 \\
4 \\
4 \\
2 \\
2 \\
1 \\
4 \\
1 \\
2 \\
28 \\
3 \\
6 \\
1 \\
2 \\
2 \\
4\end{array}$ & $\begin{array}{c}0,13 \\
2,78 \\
3,71 \\
0,13 \\
7,29 \\
1,85 \\
0,93 \\
0,79 \\
6,10 \\
4,64 \\
0,26 \\
5,83 \\
1,59 \\
0,13 \\
13,66 \\
8,09 \\
0,53 \\
0,13 \\
7,96 \\
0,26 \\
0,26 \\
0,53 \\
0,53 \\
0,26 \\
0,26 \\
0,13 \\
0,53 \\
0,13 \\
0,26 \\
3,71 \\
0,40 \\
0,79 \\
0,13 \\
0,26 \\
0,26 \\
0,53\end{array}$ & $\begin{array}{c}\text { Mega/liso } \\
\text { Noto/liso } \\
\text { Noto/liso } \\
\text { Noto/liso } \\
\text { Noto/liso } \\
\text { Noto/liso } \\
\text { Meso/liso } \\
\text { Meso/aserrado } \\
\text { Meso/aserrado } \\
\text { Noto?aserrado } \\
\text { Noto/liso } \\
\text { Meso/aserrado } \\
\text { Meso/aserrado } \\
\text { - } \\
\text { Noto/aserrado } \\
\text { Meso/aserrado } \\
\text { Micro/aserrado } \\
\text { Meso/aserrado } \\
\text { Meso/aserrado } \\
\text { Meso/aserrado } \\
\text { - } \\
\text { Meso/aserrado } \\
\text { Meso/aserrado } \\
\text { Meso/aserrado } \\
\text { Noto/aserrado } \\
\text { Noto/liso } \\
\text { Micro/liso } \\
\text { - } \\
\text { Meso/liso } \\
\text { Meso/aserrado } \\
\text { Meso/liso } \\
\text { Meso/liso } \\
\text { - } \\
\text { Noto/liso } \\
\text { Mega/liso } \\
\text { Meso/aserrado }\end{array}$ & $\begin{array}{c}\text { Pluri } \\
\text { Meso } \\
\text { Meso } \\
\text { Meso } \\
\text { Meso } \\
\text { Meso } \\
\text { Meso } \\
\text { Meso/micro } \\
\text { Meso } \\
\text { Meso } \\
\text { Meso } \\
\text { Meso/micro } \\
\text { Meso/micro } \\
\text { Meso/micro } \\
\text { Meso } \\
\text { Meso/micro } \\
\text { Meso } \\
\text { Meso y meso/micro } \\
\text { Meso/micro y micro } \\
\text { Meso/micro y micro } \\
\text { Meso/micro } \\
\text { Meso y meso/micro } \\
\text { Meso/micro } \\
\text { Meso/micro y micro } \\
\text { Meso/micro y micro } \\
\text { Meso } \\
\text { Meso? } \\
\text { Pluri } \\
\text { Meso/micro } \\
\text { Meso y meso/micro } \\
\text { Meso } \\
\text { Meso y meso/micro } \\
- \\
\text { Mega/meso } \\
\text { Mega/meso } \\
\text { Meso/micro }\end{array}$ \\
\hline $\begin{array}{l}\text { Potamogeton orbiculare } \text { Rér. } \\
\text { Arecaceae } \\
\text { Typha latissima } \mathrm{A} . \mathrm{Br} \text {. in } \mathrm{Hr} \text {. } \\
\text { Commelinidae }\end{array}$ & $\begin{array}{l}3 \\
1 \\
7 \\
13\end{array}$ & $\begin{array}{l}0,40 \\
0,13 \\
0,93 \\
1,72\end{array}$ & $\begin{array}{l}\text { Meso/liso } \\
\text { Mega/liso } \\
\text { Meso?/liso } \\
\text { Micro?/liso }\end{array}$ & $\begin{array}{l}\text { Pluri } \\
\text { Mega/meso } \\
\text { Pluri } \\
\text { Pluri }\end{array}$ \\
\hline $\begin{array}{l}\text { Dicotiledóneas indet. } \\
\text { Fruto indet. } \\
\text { Caules }\end{array}$ & $\begin{array}{l}72 \\
1 \\
3\end{array}$ & $\begin{array}{c}9,55 \\
0,13 \\
0,40\end{array}$ & $\begin{array}{l}- \\
-\end{array}$ & $\begin{array}{l}- \\
- \\
-\end{array}$ \\
\hline TOTAL RESTOS ESTUDIADOS & 754 & 100 & & \\
\hline
\end{tabular}

Figura 4. Datos macroflorísticos del afloramiento de Coll de Saig. Datos fisonómicos: Lepto: leptófilos, Micro: micrófilos, Noto: notófilos, Meso: mesófilos, Mega: megáfilos, Liso: margen foliar liso, Aserrado: margen foliar aserrado. Requerimientos térmicos: como en la Fig. 3.

La gran abundancia de elementos notófilos de tipo paleotropical de las familias Fagaceae y Lauraceae que aparece en Coll de Saig, destaca a este afloramiento del resto de los estudiados en la Cerdaña puesto que las asociaciones que presentan estos últimos se encuentran dominadas por elementos mesófilos arctoterciarios, tales 
como Quercus hispanica Rér., Alnus occidentalis, Fagus gussonii (Lám. I, fig. 10), Zelkova zelkovaefolia y Acer pyrenaicum (Lám. I, fig. 2) (Barrón, 1996a). En el afloramiento que estamos estudiando, también aparecen las especies citadas, pero en proporciones mucho menores.

Desde un punto de vista paleobiológico y tafonómico, observamos la exigua representación de las plantas démicas representadas por elementos autóctonos (Fig. 4). Únicamente se han hallado restos de Potamogeton orbiculare Rér. (Lám. I, fig. 4), Trapa ceretana Rér. y un resto foliar indeterminable de Nymphaeaceae. Una explicación de esta escasez de taxones acuáticos sería la destrucción de sus restos durante los procesos de fosilización debido a la fragilidad que por lo general poseen. A pesar de su baja representación en las asociaciones registradas del afloramiento, posiblemente las plantas acuáticas constituyeron comunidades importantes en la zona.

Las formaciones riparias y de pantano, en donde se desarrollaban los taxones démicos representados por elementos alóctonos, debieron ser importantes en la región. Aquí se integraban A. occidentalis, Ulmus sp., Salix lavateri, Populus tremulaefolia, Myrica lignitum, M. marginalis y Fraxinus numana (Lám. I, fig. 7). El componente herbáceo de estas formaciones estaría formado por Typha latissima y varios tipos de Commelinidae. Los restos mencionados como Commelinidae han sido imposibles de determinar a nivel familiar ya que podrían relacionarse de la misma forma con Poaceae, Cyperaceae, Sparganiaceae y Juncaceae.

Deducir cómo se distribuían los taxones adémicos representados por elementos alóctonos en la Cerdaña a partir de los datos macroflorísticos de Coll de Saig es bastante complicado, ya que los límites entre comunidades vegetales raramente se encuentran bien definidos en la vegetación natural y en muchos casos los ecotonos tienen una extensión mayor que las comunidades mismas (Spicer, 1981). Con respecto a la Cerdaña, Menéndez Amor (1955) expresó que la zona de ecotonía debió ser muy amplia y especialmente existente entre la vegetación de los valles y de las vertientes montañosas. Nosotros estamos de acuerdo con esta autora, ya que con seguridad los restos macroscópicos estudiados llegaron al lago desde zonas no muy alejadas y hemos encontrado en Coll de Saig restos asignables a
Lauraceae y a Tsuga que posiblemente pertenecerían a comunidades diferentes pero que seguramente habitaron en el mismo momento zonas del valle con características climáticas y topográficas similares. El estudio realizado nos permite inferir dos asociaciones diferentes:

a- Laurisilvas en las que se incluirían además de $Q$. drymeja y lauráceas, especies como Pteridium oeningense, Myrica lignitum, M. marginalis, Quercus mediterranea, Myrsiniaceae, Fabales (Lám. I, fig. 6), aff. Cedrela sp., aff. Aralia sp. y Arecaceae.

b- Bosques mixtos mesofíticos, semejantes a los que hoy en día existen en Asia Menor (Zohary, 1963; Quézel et al., 1980; Assadollahi et al., 1982) y en algunas zonas de Asia Oriental (Wolfe, 1979), en los que se desarrollaron: Zelkova zelkovaefolia, Hamamelidaceae, Fagus gussonii, Quercus hispanica, Acer pyrenaicum, Acer integerrimum, Betula insignis, Tilia vidali Rér., Cornus sp., etc..., y diferentes coníferas como Cupressaceae, Cryptomeria rhenana, Pinus sp. y Tsuga moenana.

Las condiciones climáticas que se deducen tras el estudio de los macrorrestos indican que el clima fue al menos de tipo templado ya que la mayoría de los taxones determinados son mesotérmicos o meso/microtérmicos (Fig. 4). Por su parte, el estudio fisonómico (Fig. 4) no sólo ratifica esto debido a la presencia de un 50\% de taxones mesófilos, sino que la presencia de un $19,56 \%$ de notófilos y un 6,52\% de megáfilos (siendo hasta el momento el único afloramiento de la Cerdaña en donde se han obtenido taxones megáfilos), y de un $45 \%$ de taxones con hojas de margenes enteros frente a un 55\% de hojas de márgenes aserrados, señalan que las condiciones climáticas de la zona fueron de tipo templado cálido o, posiblemente, subtropical.

\section{CONCLUSIONES: CLIMA Y VEGETACIÓN}

La flora fósil de la Cerdaña, que se desarrolló durante el Vallesiense y el Turoliense, hay que entenderla como una flora de montaña con dominancia de elementos arctoterciarios dentro de un contexto "mediterráneo", ya que la cercanía de este mar debió condicionar el clima de la zona. Gregor (1990), basándose en datos paleobotánicos, expuso que en la actual región Mediterránea, desde el Oligoceno hasta el límite Plio-Pleistoceno, se

\section{Lámina I}

Algunas especies representadas por macrorrestos en Coll de Saig (MNCNV: Museo Nacional de Ciencias Naturales, CSIC; MGM: Museo Geominero; CJQ: Colección particular Josep Querol, Sabadell). Escalas gráficas: figs. 1-2, 4-6, 8, 10-11=1 cm; fig. $3=1,5 \mathrm{~mm}$, figs. 7 y $9=5 \mathrm{~mm}$.

\section{Quercus drymeja Unger, MNCNV-4289.}

2 Acer pyrenaicum Rérolle, MGM-1076M.

3 Cupressaceae, MNCNV-713.

4 Potamogeton orbiculare Rérolle, MNCNV-4740.

5 Daphnogene sp., MGM-1074M.

6 Fabales (legumbre monosperma), CJQ-013.
7 Fraxinus numana Massalongo, MNCNV-3293.

8 Persea princeps (Heer) Schimper, MGM-227M.

9 Cryptomeria rhenana Kilpper, MNCNV-311.

10 Fagus gussonii Massalongo, MNCNV-3043.

11 Laurophyllum sp., MGM-1082M. 
Lámina I

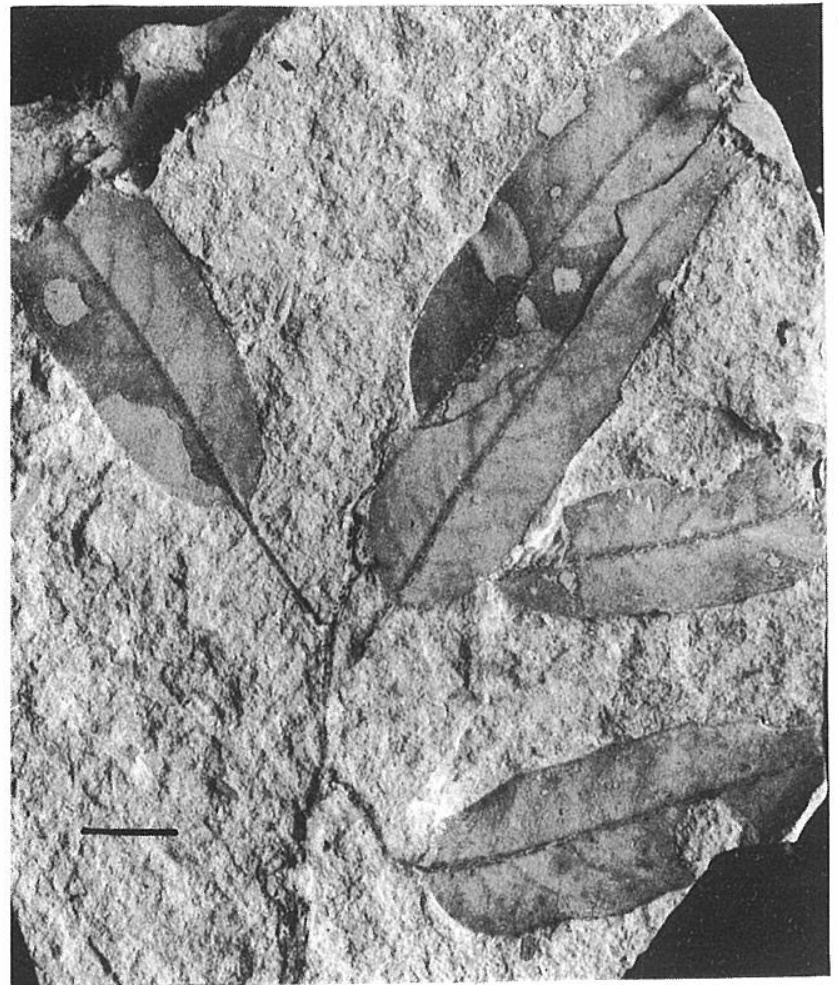

\section{1}
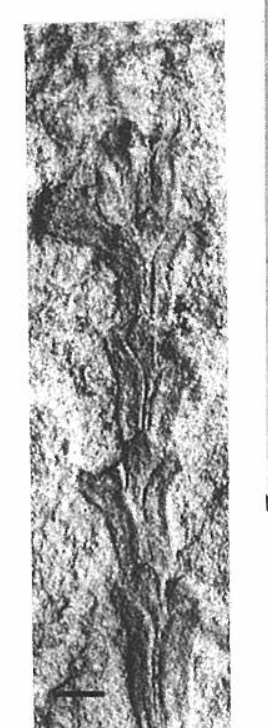

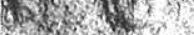
4.

3
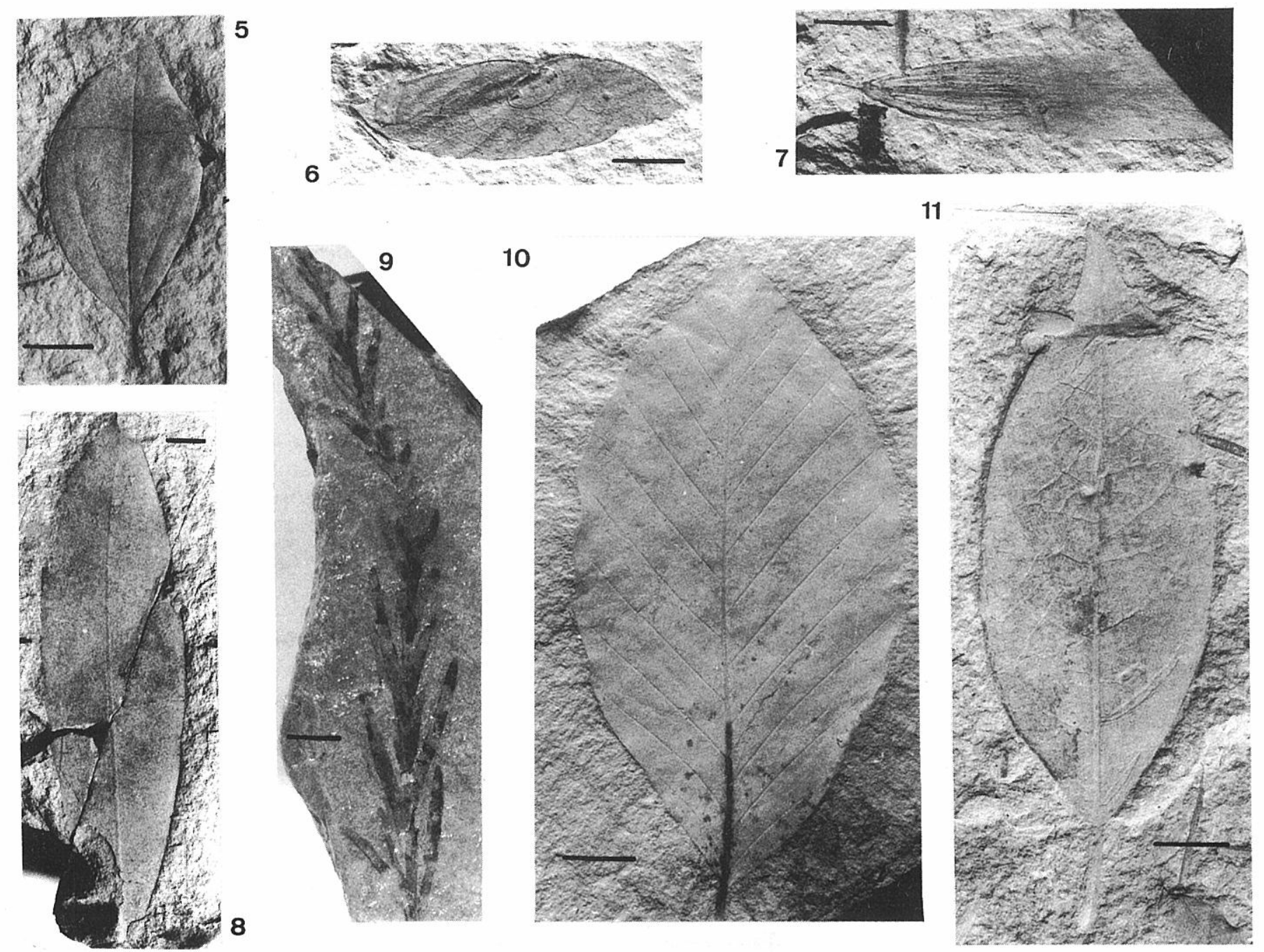

Revista Española de Paleontología, nº extr. Homenaje al Prof. J. Truyols, 1999. 
desarrolló un mesoclima de tipo templado-cálido (seguramente con microclimas particulares), con una media anual de temperaturas que oscilaba entre 13 y 19 ${ }^{\circ} \mathrm{C}$, y una media anual de precipitaciones que se encontraba entre 1.000 y $3.000 \mathrm{~mm}$.

Lo primero que se observa al estudiar el contenido paleontológico de este yacimiento es la diferencia existente entre la gran cantidad y diversidad de los restos macroscópicos recolectados y la escasez del registro polínico, que, como ya se ha explicado, es seguramente debida a la destrucción de los palinomorfos por causa de las características del afloramiento. Esto se pone de manifiesto al no haberse hallado ni un solo grano de polen atribuible a la familia Taxodiaceae (Fig. 3), siendo frecuentes en otros afloramientos de la zona (Bessedik, 1985; Barrón, 1996b, 1997). La recogida de relativamente abundantes fragmentos de ramas con hojas atribuibles a Cryptomeria rhenana (Lám. I, fig. 9), señala que la familia mencionada estuvo al menos representada por una especie en la zona.

La ausencia de pólenes de lauráceas en las muestras estudiadas resalta con los altos porcentajes de restos foliares asignables a esta familia (Figs. 3 y 4). En ninguno de los afloramientos ceretanos estudiados se ha encontrado polen de este tipo (Barrón, 1996a), lo que parece debido a la fragilidad de sus exinas (Barrón, 1996c).

Esta diferencia entre la elevada cantidad y diversidad de los restos macroscópicos estudiados y la escasez del registro polínico, así como otros factores, entre los que se encuentra la sobrerrepresentación de palinomorfos de Pinaceae, provoca que los datos micro y macroflorísticos inferidos no sean totalmente coincidentes. Esto se pone de manifiesto con respecto a las condiciones climáticas inferidas, ya que los palinomorfos las señalan de tipo templado, mientras que los macrorrestos las indican templado-cálidas o subtropicales.

Álvarez Ramis (1981, 1983), califica la flora ceretana como "montana subtropical seca". Nosotros estamos de acuerdo en los dos primeros calificativos, aunque, como hemos dicho, el clima pudo ser templado-cálido. Sin embargo, en ningún caso la flora de la Cerdaña se puede considerar seca. Los índices de precipitaciones que se debieron desarrollar en la zona fueron elevados, como se infiere al estudiar su composición paleoflorística integrada por taxones propios de laurisilvas y bosques mesofíticos. Posiblemente, el grado de humedad ambiental debió ser reseñable como también indica la presencia de laurisilvas.

Como ya hemos mencionado, el carácter montano de la flora de Coll de Saig se infiere por la presencia de taxones como Abies, el cual aparece con importantes porcentajes polínicos y posiblemente representado por piñones (Figs. 3 y 4). Esto se contradice con lo expuesto por Suc (1989), quien dijo que el polen de Abies se encuentra en la Cerdaña de una forma discreta y no aporta datos sobre la altitud de las cimas que rodeaban la zona de estudio.

Dada la cantidad de palinomorfos identificados asignables a Pinaceae, no podemos descartar que en alturas superiores al lago ceretano se desarrollara un bosque mixto de coníferas, en el sentido de Wolfe (1979). Bessedik (1985) expuso que este tipo de bosque se encontró muy bien representado a lo largo del Mioceno, y que en una cadena hipotética de vegetación, por debajo de estos bosques se desarrollaban laurisilvas. Siguiendo el razonamiento de Bessedik, y dado que Coll de Saig es el afloramiento de la Cerdaña que presenta mayor número de taxones notófilos y el único en donde se han recogido taxones megáfilos, es posible que el clima se hiciese más cálido en algunos períodos y las laurisilvas migraran altitudinalmente en la zona. Esto explicaría el tipo de vegetación subtropical del afloramiento estudiado. En este caso, los bosques mesofíticos se desarrollarían en cotas superiores, entre las laurisilvas y los bosques mixtos de coníferas.

Las apreciaciones fisonómicas que hemos realizado, combinadas con el factor de altura, puesto que la Cerdaña se encontraba a unos $1.100 \mathrm{~m}$ sobre el nivel del mar, podrían ser coincidentes con la zonación altitudinal que posee Japón (Wolfe, 1979), suponiendo que las condiciones climáticas actuales, más frías que las terciarias, han desplazado la flora del Hemisferio Norte unos cuantos grados hacia el sur. Más concretamente, si se comparan los porcentajes del tipo de margen expuestos en el apartado anterior con los que aparecen en la flora actual del este de Asia, estudiada por Wolfe (1971), concluimos que podría compararse con la flora de Taiwan, que es de tipo subtropical. De forma indicativa, señalaremos que el autor anterior da una media anual de temperaturas para bosques del Este de Asia de $19{ }^{\circ} \mathrm{C}$ y una media de rango de temperaturas de $12-19^{\circ} \mathrm{C}$.

Estos datos se apartan de lo expuesto por Álvarez Ramis (1981, 1983), en trabajos acerca de la inferencia climática a través de los datos fisonómicos de los restos foliares ceretanos, quien dio unos porcentajes muy altos para las hojas micrófilas y leptófilas, y muy bajos para las mesófilas. Pensamos que esta autora no consideró que los restos foliares recogidos en los afloramientos presentan un tamaño medio menor al que debieron poseer las hojas de los árboles que habitaban los bosques de la región, debido a una selección por tamaños durante el transporte de éstas a la zona de fosilización. A diferencia de lo expuesto por la autora citada en 1983, se han encontrado en Coll de Saig tres taxones megáfilos, lo que parece corroborar la benignidad del clima durante el depósito de los materiales de este afloramiento.

En resumen, el yacimiento de Coll de Saig destaca del resto de los afloramientos ceretanos porque ha proporcionado una macroflora muy variada, rica en elementos notófilos, siendo pobre en palinomorfos, seguramente por problemas de destrucción polínica. Durante el depósito de los sedimentos del afloramiento, en la zona se desarrollaron extensos bosques, destacándose la existencia de formaciones riparias, laurisilvas y bosques mixtos mesofíticos. Posiblemente en las cotas más altas se desarrolló un bosque mixto de coníferas. Con probabilidad, como indica la presencia de laurisilvas, el clima de la zona fue templado cálido o subtropical, semejante al que existe actualmente en 
algunas zonas de Asia Oriental, presentando un elevado régimen de precipitaciones y altos valores de humedad ambiental.

\section{AGRADECIMIENTOS}

Quiero expresar mi gratitud a Maria da Graça Nogueira Marques, por la ayuda prestada en la parte gráfica. También me gustaría destacar las importantes apreciaciones de índole científica que realizaron a este trabajo la Dra. Carmen Diéguez Jiménez y un revisor anónimo.

\section{BIBLIOGRAFÍA}

Agustí, J. y Roca, E. 1987. Síntesis bioestratigráfica de la fosa de la Cerdanya (Pirineos orientales). Estudios Geológicos, 43, 521-529.

Álvarez Ramis, C. 1981. Paleoclima de las cuencas pontienses del Pirineo catalán. In: Anais do II Congreso Latino-Americano de Paleontología, Porto Alegre, 553564.

Álvarez Ramis, C. 1983. La biometría foliar en la determinación de paleoclimas terciarios. Libro Jubilar J.M. Ríos. Contribución de Temas Generales, 3, 137-146.

Álvarez Ramis, C. y Golpe-Posse, J.M. 1981. Sobre la paleobiología de la cuenca de Cerdanya (depresiones pirenaicas). Boletín de la Real Sociedad Española de Historia Natural (Sección Geológica), 79, 31-44.

Anadón, P., Cabrera, L., Julià, R., Roca, E. and Rosell, L. 1989. Lacustrine oil-shale basins in tertiary grabens from N.E. Spain (Western European Rift System). Palaeogeography, Palaeoclimatology and Palaeoecology, 70, 7-28.

Assadollahi, F., Barbéro, M. et Quézel, P. 1982. Les écosystèmes préforestiers et forestiers de l'Iran. Ecologie Méditerranéene, 8(1-2), 365-373.

Bailey, I.W. \& Sinnot, E.W. 1915. A botanical index of Cretaceous and Tertiary climates. Science, 41, 831-834.

Bailey, I.W. \& Sinnot, E.W. 1916. The climatic distribution of certains types of angiosperm leaves. American Journal of Botany, 3, 24-39.

Baltuille, J.M., Becker-Platen, J.D., Benda, L. and Ivanovic Calzaga, Y. 1992. A contribution to the subdivision of the Neogene in Spain using Palynology. Newsletter on Stratigraphy, 27, 41-57.

Barbéro, M. et Quézel, P. 1975. Les fôrets de sapin sur le pourtoir méditerranéen. Anales del Instituto Botánico Cavanilles, 32(2), 1245-1289.

Barrón, E. 1993. Taphonomic studies of the plant remains from the ceretana Basin (Lérida, Spain). Kaupia, 2 , 127132.

Barrón, E. 1996a. Estudio tafonómico y análisis paleoecológico de la macro y microflora miocena de la cuenca de la Cerdaña. Tesis Doctoral, Facultad de Ciencias Biológicas, Universidad Complutense de Madrid, 1-773 (inédita).

Barrón, E. 1996b. Aspectos paleoecológicos de tres afloramientos de facies de abanicos aluviales del Mioceno Superior lacustre de la Cerdaña (España). In:
Estudios palinológicos (Ed. B. Ruiz Zapata). Universidad de Alcalá, Alcalá de Henares, 19-23.

Barrón, E. 1996c. Sesgos en la distribución de palinomorfos en el registro fósil. Ejemplos del lago mioceno de la Cerdaña (España). In: II Reunión de Tafonomía y fosilización (Eds. G. Meléndez Hevia, Ma F. Blasco Sancho e I. Pérez Urresti). Zaragoza, 47-54.

Barrón, E. 1996d. Caracterización del género Acer Linné (Magnoliophyta) en el Vallesiense (Neógeno) de la comarca de la Cerdaña (Lérida, España). Boletín Geológico y Minero, 107(1), 38-54.

Barrón, E. 1996e. Caracterización de la familia Betulaceae S.F. Gray (Magnoliophyta), en el Vallesiense (Neógeno) de la Cerdanya (Lleida, España). Treballs del Museu de Geologia de Barcelona, 5, 171-211.

Barrón, E. 1997. Estudio palinológico de la mina de lignito vallesiense de Sampsor (la Cerdaña, Lérida, España). Revista Española de Paleontología, 12, 91-101.

Barrón, E. 1998. Presencia del género Quercus Linné (Magnoliophyta) en el Vallesiense (Neógeno) de la Cerdaña (Lérida, España). Boletín Geológico y Minero, 109, 121-150.

Barrón, E. and Diéguez, C. 1994. Neogene species of the genus Fagus L. from la Cerdaña (Lérida, Spain). Taxonomic conclusions and phylogenetic considerations. Anales del Jardín Botánico de Madrid, 52 (1), 21-32.

Bessedik, M. 1985. Reconstitution des environnements miocènes des régions nord-ouest méditerranéennes à partir de la Palynologie. Thèse d'État. Académie de Montpellier. Univesité des Sciences et Techniques du Languedoc, Montpellier, 1-162.

Cabrera, L., Roca, E. and Santanach, P. 1988. Basin formation at the end of a strike-slip fault: the Cerdanya Basin (Eastern Pyrinees). Journal of the Geological Society of London, 145, 261-268.

Chaloner, W.G. 1968. The Paleoecology of fossil spores. In: Evolution and environments (Ed. E.T. Drake). Yale University Press, New York and London, 127-146.

Collinson, M. 1986. Use of modern generic names for plant fossils. In: Systematic and Taxonomic approaches in Palaeobotany (Eds. R.A. Spicer \& B.A. Thomas). Systematics Association, Special Volume, 31, 91-104.

Ferguson, D.K. 1974. On the taxonomy of recent and fossil species of Laurus (Lauraceae). Botanical Journal of the Linnean Society, 68, 51-72.

Fernández López, S. 1991. Taphonomic concepts for a theoretical biochronology. Revista Española de Paleontología, 6, 37-49.

Givulescu, R., Petrescu, I. et Barbu, O. 1996. Observations concernant les feuilles de Daphnogene Unger, 1845 (Lauraceae, Magnoliophyta) de l'Oligocene de Cornesti/Aghires (Département de Cluj, Roumanie). Tertiary Research, 17 (1-2), 1-4.

Green D.G. and Dolman, G.S. 1988. Fine resolution pollen analysis. Journal of Biogeography, 15, 685-701.

Gregor, H. 1990. The mesoclimatic background of the Neogene Mediterranean area reconstructed by macroflora remains. In: IX Regional Committee on Mediterranean Neogene Stratigraphy Congress, Universidad Central de Barcelona, 165-167. 
Jelgersma, S. 1957. Investigaciones palinológicas de lignitos terciarios procedentes de Cerdaña y del Valle de Arán (Pirineos españoles). Cursillos y conferencias del Instituto Lucas Mallada, 4, 159-162.

Jonker, F.P. 1951. A plea for the standarization of pollen diagrams. Taxon, 1, 89-91.

Julià, R. 1984. Síntesis geológica de la Cerdanya (Girona). In: El borde mediterráneo español: evolución del orógeno Bético y Geodinámica de las depresiones neógenas. CSIC, Granada, 95-98.

Leroy, S.A.G. and Roiron, P. 1996. Latest Pliocene pollen and leaf floras from Bernasso palaeolake (Escandorgue Massif, Hérault, France). Review of Palaeobotany and Palynology, 94, 295-328.

Martín Closas, C. 1995. Plant Taphonomy of la Cerdanya basin (Vallesian, Eastern Pyrenees). Geobios, M.S. 18, 287-298.

Menéndez Amor, J. 1955. La depresión ceretana española y sus vegetales fósiles. Característica fitopaleontológica del Neógeno de la Cerdaña española. Memorias de la Real Academia de Ciencias exactas, físicas y naturales de Madrid, serie Ciencias Naturales, 18, 1-344.

Phipps, D. and Playford, G. 1984. Laboratory techniques for the extraction of palynomorphs from sediments. Papers of the Department of Geology of the University of Queensland, 11 (1), 1-23.

Quézel, P., Barbéro, M. et Akman, Y. 1980. Contribution à l'étude de la végétation forestière d'Anatolia Septentrionale. Phytocoenologia, 8, 365-519.

Raunkiaer, C. 1934. The life forms of plants and statistical plant geography. Oxford at the Clarendon Press, Oxford, 1-632.

Rérolle, M.L. 1884-1885. Études sur les végétaux fossiles de Cerdagne. Revue des Sciences Naturelles de Montpellier, (3), 4, 167-191, 252-298, 368-386.

Roca, E. 1986. Estudi geologic de la fossa de la Cerdanya, Tesis de Licenciatura. Dpto. Geomorfología y Tectónica, Facultad de Geología, Universidad de Barcelona, 1-109 (Inédita).
Scheihing, M.H. and Pfefferkorn, H.W. 1984. The Taphonomy of land plants in the Orinoco Delta: a model for the incorporation of plant parts in clastic sediments of Late Carboniferous age of Euramerica. Review of Palaeobotany and Palynology, 41, 205-240.

Sitár, V. and Kvacek, Z. 1997. Additions and revisions to the Early Miocene flora of Lipovany (Southern Slovakia). Geologica Carpathica, 48 (4), 263-280.

Solé Sabarís, L. y Llopis Lladó, N. 1947. Mapa Geológico de España. Escala 1:50.000. Explicación de la hoja $n^{o}$ 216. Bellver.Tip. y lit. Coullaut, Madrid, 1-199.

Spicer, R.A. 1981. The sorting and deposition of allochthonous plant material in a modern environment at Silwood Lake, Silwood Park, Berkshire, England. United States Geological Survey, Proffesional Papers, 1143, 1-77.

Spicer, R.A. and Wolfe, J.A. 1987. Plant Taphonomy of the Late Holocene deposits in Trinity (Clair Engle) Lake, Northern California. Paleobiology, 13, 227-245.

Suc, J.P. 1989. Distribution latitudinale et étagement des associations végétales au Cénozoïque supérieur dans l'aire ouest méditerranéene. Bulletin de la Societé Geologique de France, (8), 5(3), 541-550.

Van Steenis, C.G.G.J. 1962. The land-bridge theory in Botany. Blumea, 11(2), 235-542.

Villalta, J.F. y Crusafont, M. 1945. La flora miocénica de la depresión de Bellver. Ilerda, 3(2), 339-353.

Webb, L.J. 1959. A physiognomic classification of Australian rain forests. Journal of Ecology, 47, 551-570.

Wolfe, J.A. 1971. Tertiary climatic fluctuations and methods of analysis of tertiary floras. Palaeogeography, Palaeoclimatology and Palaeoecology, 9, 27-57.

Wolfe, J.A. 1979. Temperature parameters of humid to mesic forests of Eastern Asia and relation to forets of other regions of the Northern Hemisphere and Australasia. United States Geological Survey, Proffesional Papers, 1106, 1-37.

Zohary, M. 1963. On the geobotanical structure of Iran. Bulletin of the Research Council of Israel, 11D suppl., 1113.

Manuscrito recibido: 26 de diciembre, 1997 Manuscrito aceptado: 16 de marzo, 1998 